\title{
FOREST REMOTE SENSING ON THE INDIVIDUAL TREE LEVEL BY AIRBORNE MILLIMETERWAVE SAR
}

\author{
Michael Schmitt ${ }^{(1)}$, Muhammad Shahzad ${ }^{(1)}$, Xiao Xiang Zhu ${ }^{(1,2)}$ \\ (1) Signal Processing in Earth Observation, Technical University of Munich (TUM), \\ Arcisstr. 21, 80333 Munich, Germany \\ (2) Remote Sensing Technology Institute (IMF), German Aerospace Center (DLR), \\ Oberpfaffenhofen, 82234 Wessling, Germany
}

\section{INTRODUCTION}

An automatic analysis of forested areas by remote sensing means is an important research topic in the context of sustainable forest management. This holds particularly for sensors and methods which are capable of providing information on the level of individual trees: In many countries, single-tree related parameters are the basis for forest inventory. Until these days, the majority of these parameters are collected manually by measurement of sample plots in cost- and time-intensive field surveys. However, remote sensing-based methods have gained increasing attention during recent decades.

A large number of promising approaches for the recognition of individual trees is based on the segmentation of $\mathrm{Li}$ DAR point clouds, e.g. [1]. When it comes to synthetic aperture radar (SAR) remote sensing, however, only recently the potential for single-tree analysis has been discovered: Beginning with the first-ever discussion of the possibilities for the recognition of individual trees by airborne millimeterwave SAR data [2], both amplitude-based and interferometric approaches have been proposed [3]. Finally, in [4] a method for the reconstruction of individual trees from multi-aspect TomoSAR data was described, while [5] presents an investigation towards the exploitation of volumetric millimeterwave SAR tomography for sub-canopy imaging. This paper summarizes the recent developments in this state-of-the-art field.

\section{MILLIMETERWAVE SAR FOR FOREST ANALYSIS}

The reason most hitherto research on SAR-based remote sensing of trees and forests used to center either on large-scale forest classification, biomass and forest volume estimation using L-band SAR tomography, or canopy height model reconstruction using X-band SAR interferometry is the rather coarse resolutions most conventional SAR sensors were able to provide to this date. In order to focus on individual trees, resolutions well in the sub-meter domain are necessary. Another reason certainly is the fact that most operational SAR systems are working in X-, C- or L-band, i.e. the long wavelengths let the signals penetrate the canopy and thus lead to a more or less significant under-estimation of the tree heights. Last, but not least, usually just one receiving antenna is mounted on carrier platforms, which means that only interferometric repeat-pass data can be collected. Since vegetation movements caused by, e.g., wind quickly lead to a decorrelation of the interferometric signals, only single-pass phase measurements allow for a reliable height reconstruction.

However, airborne single-pass millimeterwave SAR interferometry provides new access to the topic: Providing highcoherence multi-baseline interferometric SAR data, it enables the derivation of tree heights providing a convenient indicator for tree detection. Also, due to the short wavelength, it is expected to penetrate the tree canopy less than more common radar bands with longer wavelengths.

\subsection{Single-Pass SAR Tomography}

While TomoSAR basically aims at the reconstruction of continuous reflectivity profiles [6], it has also been adapted to and frequently used for discrete scenarios as they occur, e.g. in urban remote sensing $[7,8]$. Depending on the goal of tomographic analysis and on the utilized wavelength, in principle both models can be of interest for forested areas. A schematic sketch of the TomoSAR configuration for forested areas, comparing both the continuous and the sparse reflectifity hypotheses is presented in Fig. 1. For display purposes, only logarithmic amplitudes are shown in the remainder of this paper.

\subsubsection{SAR Tomography for Continuous Reflectivity Profiles}

For sake of simplicity, the well-known spectral estimator MUSIC (multiple signal classification) [9] is used for the evaluation of the millimeterwave test data aiming at continuous reflectivity profiles. For TomoSAR inversion, the 


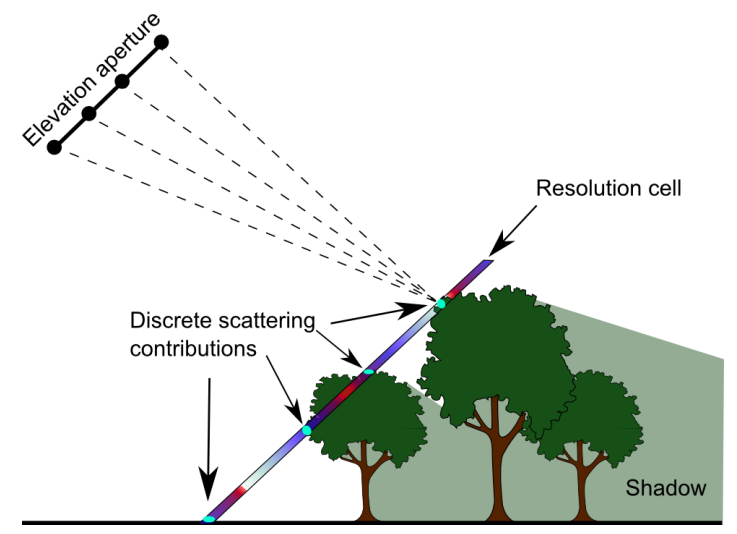

Fig. 1. Sketch of the TomoSAR acquisition geometry for forested areas. If the continous reflectivity hypothesis is used, the whole reflectivity profile of the resolution cell is reconstructed and will probably show stronger reflectivities at or inside tree structures. In contrast, if the discrete reflectivity hypothesis is used, only discrete scattering contributions at the sensor-facing tree structures are expected.

so-called MUSIC pseudo-spectrum

$$
P_{M U S I C}(h)=\frac{1}{\mathbf{a}^{H}(h) \mathbf{E}_{n}^{H} \mathbf{E}_{n} \mathbf{a}(h)}
$$

is calculated, where a $(h)$ is the so-called steering vector corresponding to a scattering contribution expected at height $h$, and $\mathbf{E}_{n}$ is the matrix containing the eigenvectors corresponding to the noise subspace.

\subsubsection{SAR Tomography for Discrete Reflectivity Profiles}

In order to evaluate the discrete scattering hypothesis for millimeterwave-based SAR tomography of forest volumes, the maximum-likelihood TomoSAR (ML-TomoSAR) approach proposed in [8] is employed for TomoSAR point cloud generation.

In contrast to conventional spectral inversion methods, here a multi-dimensional objective function

$$
\hat{\mathbf{h}}=\underset{\mathbf{h} \in\left[h_{\text {min }} ; h_{\text {max }}\right]}{\arg \max } \exp \left(-\tilde{\mathbf{z}}^{H}(\mathbf{h}) \tilde{\mathbf{C}}^{-1} \tilde{\mathbf{z}}(\mathbf{h})\right),
$$

where $\tilde{\mathbf{C}}$ is the sample coherence matrix of a resolution cell, is optimized in order to find the most likely combination of up to $N-1$ scatterers (where $N$ is the number of receiving antennas of the multi-baseline system). Instead of the one-dimensional search with following peak detection that usually is employed if MUSIC is used for the reconstruction of discrete scattering profiles, here a $K$-dimensional optimization for the unknown scatterer heights is solved by a multi-dimensional grid search. This is realized by not using a simple steering vector as $\mathbf{a}(h)$ in (1), but a so-called model signal vector $\tilde{\mathbf{z}}(\mathbf{h})$, which describes the signal expected from a mixture of $K$ scattering contributions at heights $\mathbf{h}=\left[h_{1}, \ldots, h_{K}\right]^{T}$.

\subsection{Single-Tree Reconstruction from TomoSAR Point Clouds}

\subsubsection{Multi-Aspect TomoSAR Data Fusion}

The TomoSAR post-processing necessary for the reconstruction of individual trees is described in [4]: After 3D point clouds in slant range geometry have been reconstructed for TomoSAR data of different viewing directions (i.e. aspects), the data have to be geocoded and projected to a common world coordinate system. Then, the different point clouds are fused to a joint point cloud in order to mitigate data gaps caused by radar shadow. Besides, the amount of point data is significantly reduced and a certain amount of denoising is achieved this way.

\subsubsection{Mean Shift Clustering}

In order to reconstruct individual trees, the final multi-aspect 3D TomoSAR point cloud is clustered by the mean shift algorithm as described by [10]. Since the tree crowns generally show a comparably high point density, the points are clustered in the spatial domain, i.e. the feature space is comprised of spatial coordinates in Euclidian space. Mean shift clustering essentially seeks modes of the kernel density estimates and works iteratively by shifting every data point toward the weighted mean of points within its neighborhood (defined to be cylindrical in the presented case). The shift vector $\mathbf{m}\left(\mathbf{p}_{i}\right)$ always points towards the direction of the maximum increase in the kernel density $D_{\mathbf{p}_{i}}$ and is computed as

$$
\mathbf{m}\left(\mathbf{p}_{i}\right)=\frac{\sum_{j=1}^{n} \mathbf{p}_{j} \exp \left(-\frac{\left\|\mathbf{p}_{i}-\mathbf{p}_{j}\right\|^{2}}{b^{2}}\right)}{\sum_{j=1}^{n} \exp \left(-\frac{\left\|\mathbf{p}_{i}-\mathbf{p}_{j}\right\|^{2}}{b^{2}}\right)}-\mathbf{p}_{i} .
$$

The iteration process continues until there is no or only little shift in $\mathbf{m}\left(\mathbf{p}_{i}\right)$ anymore, i.e. the length of the shift vector $\mathbf{m}\left(\mathbf{p}_{i}\right)$ is close to 0 . Due to the gradient ascent nature, the mean shift algorithm returns clusters using the concept attraction of basin, i.e. those points whose trajectories lead to the same mode form the basin of attraction for that mode and are clustered into one group. The clustering procedure is repeated until all points are assigned to their respective modes.

\subsubsection{Robust Modeling of Ellipsoidic Tree Crowns}

Since three-dimensional rotational ellipsoids can be seen as a good approximation of deciduous tree crowns, the individual tree clusters are modeled using generalized tri-axial ellipsoids. For this purpose, parameters of an arbitrarily oriented minimum volume enclosing ellipse (MVEE) are estimated by first projecting points belonging to individual tree clusters onto the $x y$-plane followed by extruding the 2D $x y$ ellipse in $z$-direction to form a 3D ellipsoid. The motivation for expanding the ellipsoid along the $z$-axis is based on geometrical considerations: It is assumed that correct tree models 
may have an arbitrary orientation in the $x y$-plane, but remain upright or vertical with respect to the ground. This is based on the light prior that tree trunks are modeled to be vertical to the ground surface.

\section{EXPERIMENTS AND RESULTS}

\subsection{Test Data}

The sensor used for the experimental considerations in this paper is the German MEMPHIS system created by the Fraunhofer FHR [11]. Although it is able to be operated in different modes and configurations, in this work only the basic airborne side-looking configuration with a carrier frequency of $35 \mathrm{GHz}$ (Ka-band) and a bandwidth of $900 \mathrm{MHz}$ is employed. This leads to a slant range resolution of $16.7 \mathrm{~cm}$, whereas the azimuth resolution is $5.2 \mathrm{~cm}$. Since MEMPHIS is equipped with four receiving antennas, it is able to provide multi-baseline InSAR datasets from just a single pass over the scene of interest.

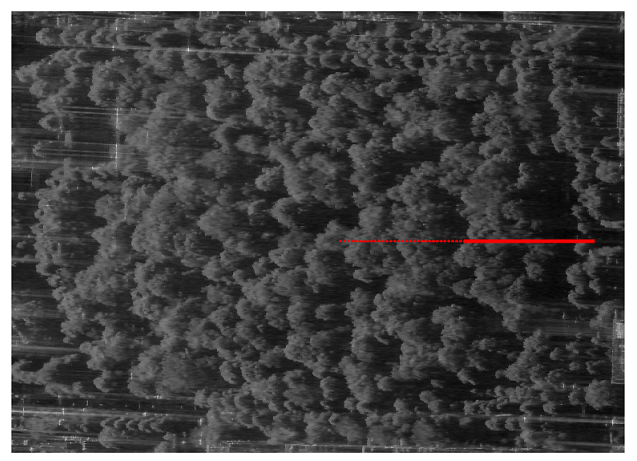

Fig. 2. SAR intensity image of the test scene (SAR range direction from left to right). The red line shows the investigated TomoSAR profiles.

The available experimental MEMPHIS data were acquired during a campaign over Munich, Germany, in 2013. The test scene contains the "Alter Nordfriedhof", an abandoned cemetery, which is used as a public park today. As can be seen in Fig. 2, it is mainly characterized by a light planting of trees, resembling a grove or little wood.

\subsection{Volume Tomography Results}

Figure 3 (a) shows the tomographic slices corresponding to the solid line in Fig. 2, processed with MUSIC for a fixed model order of $K=3$ (corresponding to the maximum degrees of freedom of a 4-antenna system such as MEMPHIS). Correspondingly, Fig. 3 (b) shows LiDAR ground truth projected into radar geometry. A different perspective is given in Fig. 4, where additionally a MUSIC tomogram with automatic model order selection is provided. It is extended by some range bins towards the sensor (marked by the dashed line in Fig. 2), discretized and geocoded as point cloud. This way, the tomographic data can be overlayed with the LiDAR ground truth in world geometry. From both the tomographic slices shown in Fig. 3 and the geocoded tomogram in Fig. 4, it can be seen that generally the main responses are located at the tree crowns facing the sensor. In addition, it is noticeable that the tomographic data contains more information related to subcanopy scene content: As the white box in Fig. 4 indicates, ground structures also exhibit a significant amount of backscatter, although actually covered by larger tree crowns. This indicates that millimeterwave SAR provides a certain amount of canopy penetration at least if the canopy is not too dense.

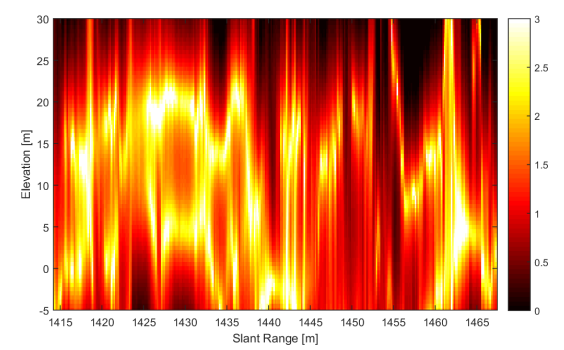

(a)

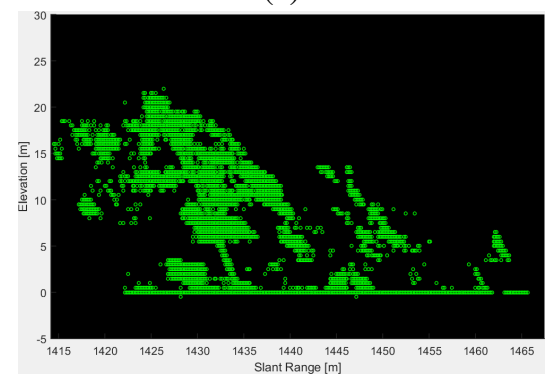

(b)

Fig. 3. (a) MUSIC tomogram with model order $K=3$, (b) LiDAR ground truth projected into radar geometry).

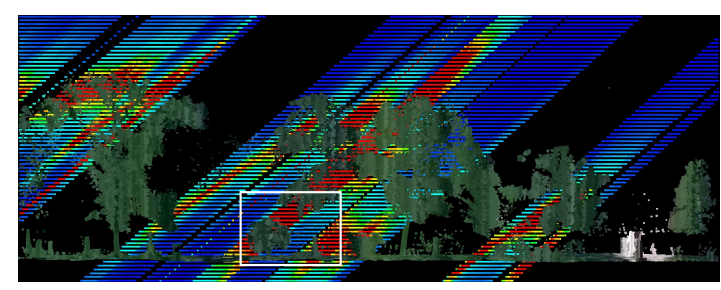

Fig. 4. Geocoded MUSIC tomogram with model order $K=2$, overlayed onto LiDAR ground truth. The white box indicates strong subcanopy backscattering.

\subsection{Single-Tree Reconstruction Results}

The result of the discrete TomoSAR processing by the MLTomoSAR algorithm and subsequent multi-aspect point cloud 
fusion is shown in Fig. 5 (a); the result of the mean shift clustering of the resulting point cloud with a bandwidth parameter of $3.2 \mathrm{~m}$ is displayed in Fig. 5 (b). It can be seen that the clusters already resemble individual trees by visual impression. The final result of the ellipsoid modeling process can be assessed in Fig. 5 (c), including tree crowns of different shape and hypothetical stem positions.

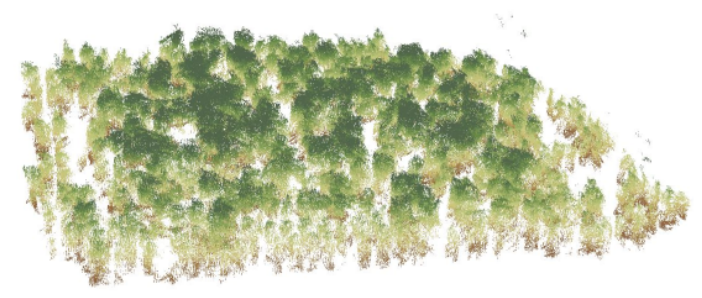

(a)

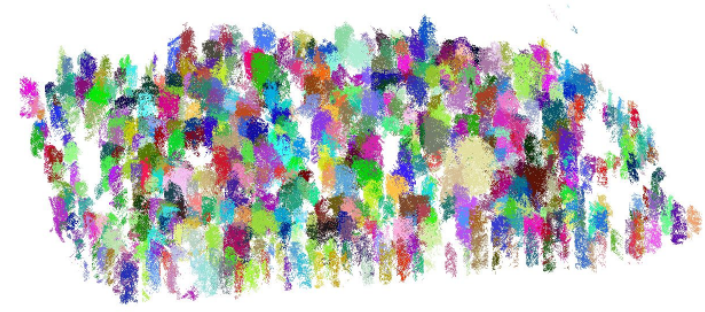

(b)

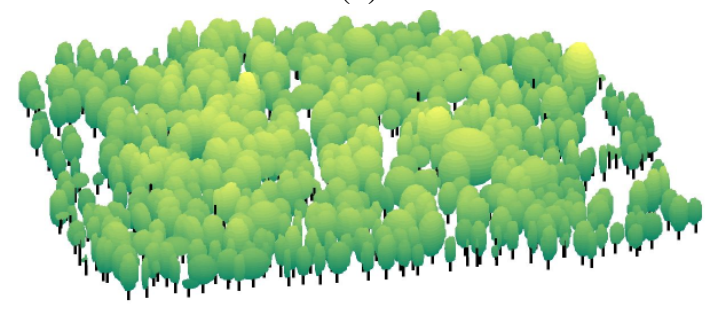

(c)

Fig. 5. The scene shown in different processing stages: (a) The 3D point cloud as derived by multi-aspect TomoSAR data fusion; (b) the clustered point cloud; (c) the reconstructed tree models.

\section{CONCLUSION}

This paper presented experimental results discussing the potential of millimeterwave SAR for forest remote sensing on the individual tree level. As can be seen from the experimental results, although there is a certain amount of canopy penetration, a significant part of the signal response is received from the tree crowns. This provides both interesting perspectives for an analysis of forest volumes by continuous TomoSAR models as well as the reconstruction of individual tree models by utilization of discrete TomoSAR models.

\section{ACKNOWLEDGMENT}

The authors would like to express their thanks to S. Stanko and T. Brehm of Fraunhofer FHR as well as E. Meier and C. Magnard of RSL Zurich for providing the MEMPHIS test data. This work is supported by the Helmholtz Association under the framework of the Young Investigators Group "SiPEO (VH-NG-1018, www.sipeo.bgu.tum.de).

\section{REFERENCES}

[1] Josef Reitberger, Claudius Schnörr, Peter Krzystek, and Uwe Stilla, "3D segmentation of single trees exploiting full waveform LIDAR data," ISPRS J. Photogramm., vol. 64, no. 6, pp. 561-574, 2009.

[2] Michael Schmitt, Axel Brück, Johannes Schönberger, and Uwe Stilla, "Potential of airborne single-pass millimeterwave InSAR data for individual tree recognition," in Proc. 33. Jahrestagung der DGPF, 2013, pp. 427-436.

[3] Uwe Stilla, Michael Schmitt, Oliver Maksymiuk, and Stefan Auer, "Towards the recognition of individual trees in decimeter-resolution airborne millimeterwave SAR," in Proceedings of 8th IAPR Workshop on Pattern Recognition in Remote Sensing, 2014, on CD.

[4] Michael Schmitt, Muhammad Shahzad, and Xiaoxiang Zhu, "Reconstruction of individual trees from multi-aspect TomoSAR data," Remote Sensing of Environment, vol. 165, pp. 175-185, 2015.

[5] Michael Schmitt and Xiaoxiang Zhu, "Demonstration of single-pass millimeterwave SAR tomography for forest volumes," IEEE Geoscience and Remote Sensing Letters, vol. 13, no. 2, pp. 202-206, 2016.

[6] Andreas Reigber and Alberto Moreira, "First demonstration of airborne SAR tomography using multibaseline L-band data," IEEE Transactions on Geoscience and Remote Sensing, vol. 38, no. 5, pp. 2142-2152, 2000.

[7] Xiaoxiang Zhu and Richard Bamler, "Tomographic SAR inversion by L1-norm regularization - The compressive sensing approach," IEEE Transactions on Geoscience and Remote Sensing, vol. 48, no. 10, pp. 3839-3846, 2010.

[8] Michael Schmitt and Uwe Stilla, "Maximum-likelihood-based approach for single-pass synthetic aperture radar tomography over urban areas," IET Radar, Sonar \& Navigation, vol. 8, no. 9, pp. 1145-1153, 2014.

[9] Ralph O. Schmidt, "Multiple emitter location and signal parameter estimation," IEEE Transactions on Antennas and Propagation, vol. 34, no. 3, pp. 276-280, 1986.

[10] D. Comaniciu and P. Meer, "Mean shift: a robust approach toward feature space analysis," IEEE Transactions on Pattern Analysis and Machine Intelligence, vol. 24, no. 5, pp. 603-619, 2002.

[11] H. Schimpf, Helmut Essen, S. Boehmsdorff, and T. Brehm, "MEMPHIS - a fully polarimetric experimental radar," in Proceedings of IEEE International Geoscience and Remote Sensing Symposium, 2002, pp. 1714-1716. 